\title{
Correction to: Regional disparity of medical resources and its effect on age-standardized mortality rates in Korea
}

\author{
Insu Chang ${ }^{1}$ Brian H. S. Kim²
}

Published online: 15 July 2019

๑) Springer-Verlag GmbH Germany, part of Springer Nature 2019

\section{Correction to: The Annals of Regional Science (2019) 62:305-325 https://doi.org/10.1007/s00168-019-00897-z}

The original version of the article unfortunately contained an error in Acknowledgements section. Below is the corrected version.

Acknowledgements This work was supported by the Ministry of Education of the Republic of Korea and the National Research Foundation of Korea (NRF-2017S1A3A2066771).

The original article can be found online at https://doi.org/10.1007/s00168-019-00897-z.

Brian H. S. Kim

briankim66@snu.ac.kr

1 Department of Population Policy Research, Korea Institute for Health and Social Affairs, Building D, 370 Sicheong-daero, Sejong City 30147, Korea

2 Program in Regional Information, Department of Agricultural Economics and Rural Development, Program in Agricultural and Forest Meteorology, Research Institute of Agriculture and Life Sciences, Seoul National University, 1 Gwanak-ro, Gwanak-gu, Seoul 08826, Korea 